\title{
Array of lenses with individually tunable focal-length based on transparent ion-implanted EAPs
}

\author{
Muhamed Niklaus, Samuel Rosset ${ }^{+}$, Herbert Shea $^{*}$ \\ Ecole Polytechnique Fédérale de Lausanne (EPFL), rue Jaquet-Droz 1, 2002 Neuchâtel, \\ Switzerland
}

\begin{abstract}
We report on the fabrication and characterization of 2x2 arrays of mm-diameter PDMS lenses whose focal length can be electrically tuned. Dielectric elastomer actuators generally rely on carbon powder or carbon grease electrodes, which are not transparent, precluding the polymer actuator from also being a lens. However compliant electrodes fabricated by low-energy ion implantation are over 50\% transparent in the visible, enabling the polymer lens to simultaneously be an actuator. We have developed a chip-scale process to microfabricate lens arrays, consisting of a molded socket bonded to a Pyrex chip supporting 4 membrane actuators. The actuators are interconnected via an incompressible fluid. The Pyrex chip has four through-holes, 1 to $3 \mathrm{~mm}$ in diameter, on which a $30 \mu \mathrm{m}$ thick Polydimethysiloxane (PDMS) layer is bonded. The PDMS layer is implanted on both sides with $5 \mathrm{keV}$ gold ions to define the transparent electrodes for EAP actuation. Applying a voltage to one of the lens/actuators leads to an area expansion and hence to a change in radius of curvature, varying the focal length. We report tuning the focal length from $4 \mathrm{~mm}$ to $8 \mathrm{~mm}$ at $1.7 \mathrm{kV}$, and present changes in optical transmission and membrane stiffness following gamma and proton irradiation.
\end{abstract}

Keywords: Metal ion implantation, Dielectric elastomer actuator, Polydimethylsiloxane, Lenses

\section{INTRODUCTION}

Tunable micro-optical elements are an active field of research, with applications in optical sensing, beam steering, beam shaping, endoscopes, and laser trapping. ${ }^{1}$ Microlens arrays are now commonly found in chip-scale cameras, in Shack-Hartmann type wavefront sensors, and for many laser applications.

PDMS (Polydimethylsiloxane) is a polymer that can offer excellent optical properties: transparent from near-UV to near-IR, flexible, stable over a large temperature range, low surface roughness if spin-coated, index of refraction of 1.4. It is commonly used for replication and is compatible with a range of microfabrication techniques. Several groups have developed arrays of identical PDMS lenses with a fixed focal length that can be chosen within a large range during microfabrication without requiring new lithography masks. For instance Grilli et al. use periodically poled lithium niobate crystals whose surface wettability depends on the electric field to generate arrays of identical PDMS microlenses. $^{2}$ Hongbin et al. and Zeng et al. developed a process to fabricate arrays of PDMS lenses, whose focal length, though fixed after curing, can be set during microfabrication through pneumatic control., ${ }^{3,4}$

Several group have reported the focal length tuning of single PDMS lenses, using electroactive polymers, pneumatic (analog and digital) and thermal. ${ }^{5-8}$ Fewer publications concern lens arrays whose focal length can be tuned during operation. Zappe's group has reported pneumatic tuning of PDMS lens arrays. ${ }^{9}{ }^{10}$ The technique is effective, but all lenses move simultaneously. The technique is aimed at small arrays or single lenses, and allows for low voltage operation when coupled with their thermo-pneumatic actuator.

\footnotetext{
*herbert.shea@epfl.ch, EPFL-LMTS, rue Jaquet-Droz 1, CP 526, 2002 Neuchâtel, Switzerland, tel +4132 720 5584, http://lmts.epfl.ch/

${ }^{+}$now with OptoTune SA, Zurich, Switzerland
}

Electroactive Polymer Actuators and Devices (EAPAD) 2010, edited by Yoseph Bar-Cohen, Proc. of SPIE Vol. 7642, 76422K · (c) 2010 SPIE · CCC code: 0277-786X/10/\$18 · doi: 10.1117/12.848445 
Tuning individual lenses within a large array cannot be done practically using pneumatic control because of the complex fluidic network with hundreds of pressure controls that would be needed. Currently, only electrowetting allows individual tuning electrically, but liquid lenses bring many difficulties compared to PDMS lenses. Having arrays of individually tunable lenses would be a powerful tool for adaptive optics, optical switching, imaging, laser trapping, and for biological applications or for quantum computing, allowing trap position to be dynamically changed for individual cells or atoms.

PDMS is commonly used as the elastomer in Dielectric Elastomer Actuator (DEA), an emerging class of actuators that have demonstrated high efficiency and large displacement (10\% to $100 \%$ elongation) for macro-scale ( $\mathrm{cm}$ to $\mathrm{m}$ ) devices, often referred to as artificial muscles. ${ }^{11,12}$ Efforts at miniaturizing DEAs have been mostly hindered by the lack of a microfabrication-compatible technique to make compliant electrodes that can: 1) conduct at repeated strains of greater than $50 \%, 2$ ) be patterned on the micron scale, and 3) not stiffen the polymer. Satisfying all three requirements simultaneously is challenging, since metals have Young's modulus five orders of magnitude larger than polymers, and metal films crack at strains of greater than approximately 3\%. For macroscale EAP devices, the solution of choice has been carbon powder, either painted on, mixed with grease, or mixed with the unpolymerized elastomer. ${ }^{13-15}$

Our laboratory has developed a technique to microfabricate highly compliant electrodes in/on polymers using metal ion implantation at low energies (1 to $5 \mathrm{keV}$ ) and with that technology we fabricate DEA micro-actuators whose efficiency is the same as macro-scale artificial muscles. ${ }^{16,17}$ The implantation process creates a nano-composite a few $\mathrm{nm}$ thick, located in the top tens of $\mathrm{nm}$ of the surface of the elastomer. ${ }^{18}$ The implanted dose must be high enough to obtain a conductive layer (e.g., by percolation between nanoparticles), but low enough not to affect the mechanical properties of a metallic film. This technique allows the $\mu \mathrm{m}$ scale patterning of conductive electrodes that do not significantly stiffen thin PDSM membranes, yet conduct at over $150 \%$ strain. $^{16}$

A unique aspect of the metal-ion implanted electrodes is their optical transparency, opening the possibility of making transmissive optical devices using PDMS DEA actuators, with high fill factors and actuators with features patterned with down to $20 \mu \mathrm{m}$ resolutions. ${ }^{16}$

In this article we report on a proof-of-concept study of tunable micro-optical components based on DEAs. We have developed polymer tunable micro-lenses coupled with DEA by means of water filled channels allowing a transfer of the actuation to a variation of focal lengths. This design takes advantage of the good optical transmission of PDMS (90\% in the visible when not implanted, 50\% when implanted). Each lens has either its own individually addressed DEA or many of them dependent on the needed performances and applications. A tuning range of a factor of 2 in focal length is experimentally observed. Here we describe the design, fabrication, tests, performance and modeling of tunable micro-optical lenses.

\section{DESIGN AND FABRICATION}

The design and fabrication of our devices are driven by the following goals:

- Lens diameter: the approach should enable lenses and actuators of diameter $100 \mu \mathrm{m}$ to $5 \mathrm{~mm}$.

- Array: the small lens diameter combined with individual addressing enables to address independently many actuators on fabricated on one substrate.

- Focal length tuning range should be as large as possible.

- Optical properties: PDMS is a material that has already been reported being used in optical devices. Hence very good optical properties are expected, subject to good surface roughness control.

- Robustness: the monolithic technology proposed should be more robust than existing actuators that have an actuation mechanism external to the optical component.

- Space qualification potential: PDMS has a glass transition temperature of around $-135^{\circ} \mathrm{C}$ and can easily be heated up above several hundreds of Celsius, which makes it suitable for many space applications. 


\subsection{Design}

The tunable polymer micro-optical device consists of a fluid (water or oil) encapsulated into a deformable cavity sealed by PDMS membranes, which can serve as lenses or as actuators (Figure 1). The initial fluid pressure is such that all of the membranes at the beginning are bulging outwards (convex). The membranes have compliant electrodes on both sides, which allow an electrostatic force to be applied, which compresses the membranes, increasing it's area, and hence deforming due to the liquid pressure. When one membrane extends, any coupled membrane will contract. Consequently the focal lengths of the lenses change (Figure 2). Different connection configurations between the lenses and actuators lead to various scales of focal length (one or more of DEAs connected with one or more lenses, see Figure 3). The system thus works in a push-pull configuration. For simplicity, we shall focus on a system, as in Figure 2, with one active devices (DEA membrane, the "actuator") and one passive device (PDMS membrane, the "lens").

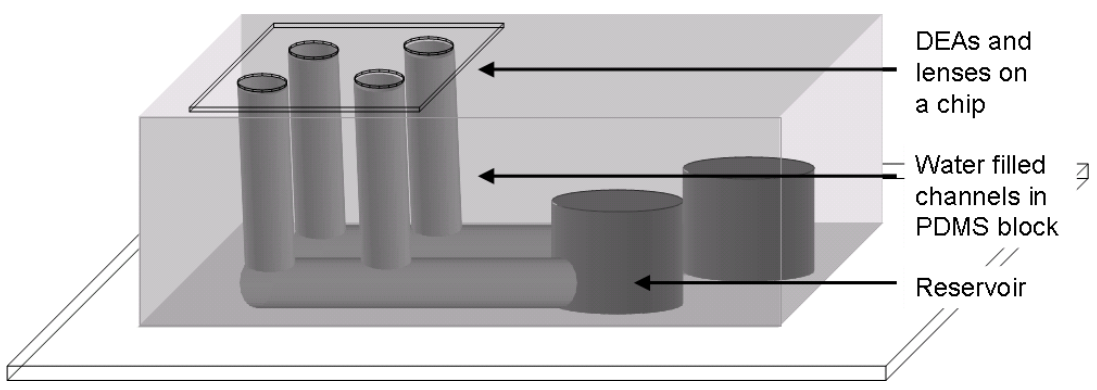

Figure 1. Schematic view of the tunable lens device. A chip with four polymer membranes (actuators and lenses) is bonded on a PDMS block, in which communicating channels and reservoirs have been filled with water. Light passes vertically through the system. The radius of curvature of the membranes can be electrically tuned.

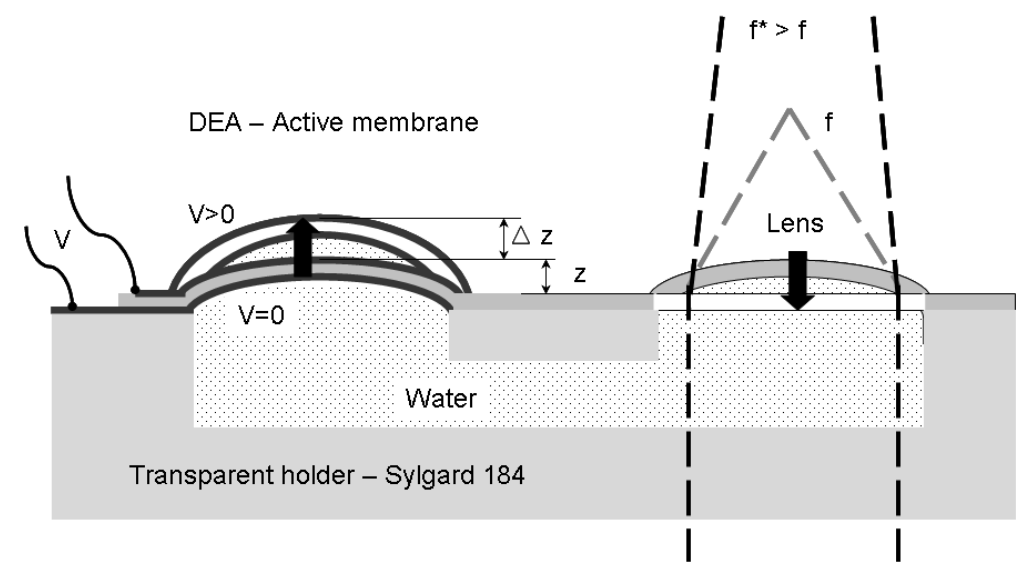

Figure 2: Principle of operation: applying a voltage to an actuator (the active membrane on the left) increases its area, leading to a larger radius of curvature. This reduces the radius of curvature of the lens (non-actuated membrane on the right), to which it is coupled through the channels, and increases the focal length $f$ of the right membrane, while decreasing the focal length of the left membrane.

The tunable lens system consists of the two main parts:

- Chip with PDMS actuators and lenses: consisting of a through-hole Pyrex chip $\left(2 \times 2 \mathrm{~cm}^{2}\right)$ with a soft PDMS (Sylgard 186, Dow Corning, $1 \mathrm{MPa}$ Young's modulus) film $(20-40 \mu \mathrm{m})$ on the top. There are compliant implanted electrodes on the top and bottom of the membrane, as well as wires for connections to power supplies.

- Transparent holder: a $7.5 \times 2.5 \times 0.5 \mathrm{~cm}^{3}$ block molded from of a harder PDMS (Sylgard 184, Dow Corning) containing fluidic channels and reservoirs. The latter are needed in order to control the water pressure in the channels. 


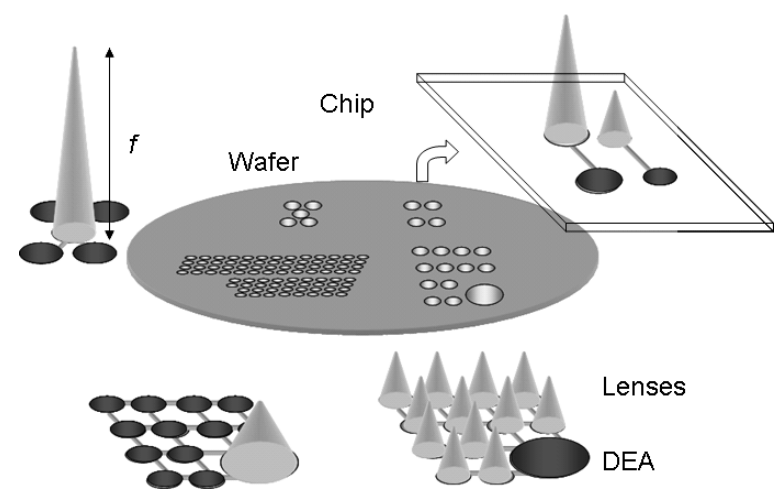

Figure 3. Some possible device configurations of lenses and DEAs (darkest circles) on a wafer scale or a chip scale level, showing the broader range of tuning configurations than the simple one illustrated in Figure 2.

\subsection{Fabrication}

One of the key factors to obtain large displacements with DEAs is to have compliant electrodes. Most of macro-sized DEAs have electrodes made of conducting grease or powder (metal, graphite...). This method is not applicable to miniaturized DEAs for which the electrodes must be patterned on the micron-scale, in order to have many independently-addressable actuators. Standard clean-room compatible electrode creation methods, such as metal evaporation, tend to greatly increase the actuator's rigidity, which negatively affects its performance. ${ }^{19}$

Our laboratory has introduced low-energy ion-implantation to create highly compliant electrodes, with which we have made mm-size diaphragm actuators with vertical displacement of more than $25 \%$ of the membrane's diameter, with response times less than $1 \mathrm{~ms}$, and RC time constants much shorter than mechanical time constants (see Figure 4). ${ }^{20,21}$
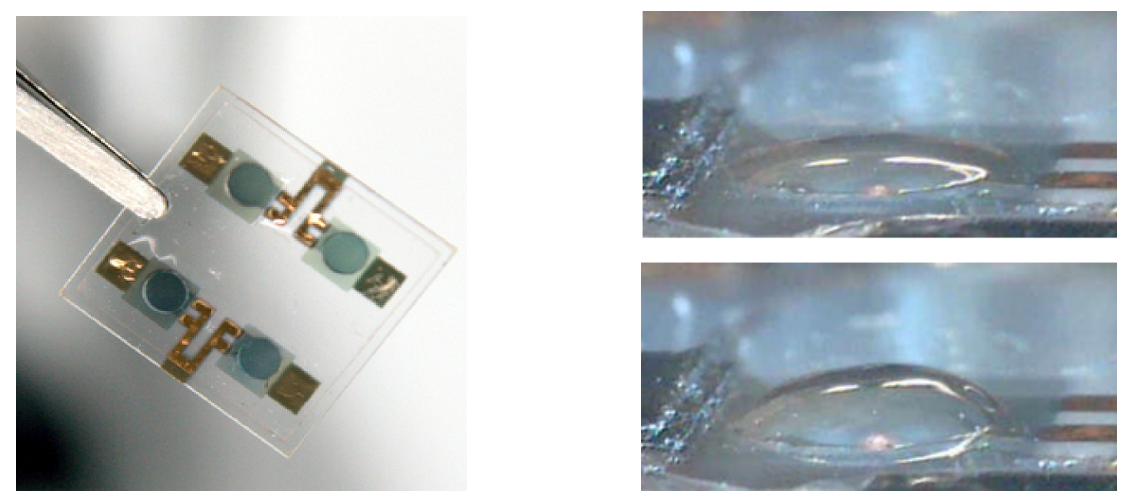

Figure 4. Diaphragm actuators. Left: Four DEAs $(\varnothing 3 \mathrm{~mm})$ on $2 \times 2 \mathrm{~cm}^{2}$ chip. The fabrication steps are: 1 . Implantation of gold electrodes into $30 \mu \mathrm{m}$ thick PDMS film and deposition of Au contacts (bottom implantation); 2. Deposition of Au contacts on a 2x2 $\mathrm{cm}^{2}$ through-hole $(\varnothing 1-3 \mathrm{~mm})$ Pyrex chip, aligning and bonding together with the implanted PDMS; 3 . Top implantation and deposition of Au contacts. Right: A $3 \mathrm{~mm}$ diameter actuator at $0 \mathrm{~V}$ (top image) and $1600 \mathrm{~V}$ (bottom image). The membrane is not flat at $0 \mathrm{~V}$ because of an initial pressure of $125 \mathrm{~Pa}$ was applied to promote upwards displacement.

The fabrication procedure of DEAs using ion-implanted PDMS films has been explained in details in our earlier publications (see the caption of Figure 4 for a short summary). ${ }^{22}$ Hence this proceeding contains only the further development related to the tunable lenses.

Lenses are made in the same way as the DEAs, but skipping the steps with implantation and deposition of gold in order to maximize optical transmission, and minimize surface roughness. Non-polymerized PDMS is spin-coated to 30 
$\mu \mathrm{m}$ on a Polyimide sheet, heated at $100^{\circ} \mathrm{C}$ during 30 minutes to cure, and bonded onto a through-hole Pyrex chip which determines the diameter of the membranes. The lenses can be made separately on another chip or by using a shadow mask if on the same chip as DEAs (to avoid implanting the lenses). In the first case lenses can be made with completely different properties than DEAs, which might be useful as it adds flexibility in the choice of films mechanical properties.

The transparent holder ( $\left.5 \times 2.5 \times 0.5 \mathrm{~cm}^{3}\right)$ is molded from PDMS (Sylgard 184) and contains different number of channels depending on the chosen configuration and functioning scale of lenses. The block also includes one or more cavities that serves as reservoirs after the filling. They are necessary to adjust the initial pressure of the fluid to set the operating point.

\subsection{Assembly}

The optical device chip containing DEAs and lenses is bonded on the top of the PDMS support (sealing of the top). The assembly is turned upside down and filled with a fluid paying attention that the fluid wets the membranes well without bubbles. The bottom is sealed with a glass plate $\left(5 \times 2.5 \mathrm{~cm}^{2}\right)$. If necessary, additional corrections of the fluidic volume can be achieved with a syringe penetrating directly through the PDMS block. The electrical wires were glued to the deposited gold contacts on the chip.

\section{EXPERIMENTAL METHODS}

\subsection{Irradiation}

Both ion-implanted and virgin PDMS membranes were tested for optical transmission and mechanical properties before and after irradiation. The samples consisted of a Pyrex chip with the $30 \mu \mathrm{m}$ PDMS Sylgard 186 membrane, either with (DEA) or without (lens) ion-implanted electrodes.

For the optical transmission tests we measured the transmission directly with a spectrometer (Ocean Optics usb4000). The mechanical properties were obtained by fixing the optical devices on a sealed socket and by applying air pressure $P$ on the lenses or the DEAs. This forces the membranes to bulge and the relation between the vertical displacement and the pressure allows us to extract the Young's modulus and internal stress, known as a bulge test. ${ }^{23}$

The irradiation were performed by the University of Padova. We have analyzed the influence of the absorbing dose i.e., total ionizing dose (TID) on the Young's modulus and on the residual stress. The gamma (Co60) irradiation was performed with two different dose rates: $540 \mathrm{~Gy} / \mathrm{hr}\left(\right.$ in $\left.\mathrm{H}_{2} \mathrm{O}\right)$ and $29 \mathrm{~Gy} / \mathrm{hr}\left(\mathrm{in}_{2} \mathrm{O}\right)$; this in order to establish also the dependence of the properties on the intensity of the radiation. The correction factor taken for silicon was about 0.9 . The final doses were $11840 \mathrm{~Gy}, 572$ Gy (during 18 hours) and 594 Gy (during 1.1 hours).

The proton irradiation was performed at $3.5 \mathrm{MeV}$, with the dose rate of about $690 \mathrm{pA}$, and at the three doses: $1 \mathrm{kGy}, 5$ kGy and $10 \mathrm{kGy}$.

\subsection{Focal length}

For the tests of the performance of the final device DEAs were actuated with a voltage of $1.7 \mathrm{kV}$ and the liquid used inside channels was water. The configuration of the final device used was one DEA changing the focal length of one lens, as in Figure 2.

The focal length of the lens was measured as follows: A collimated laser beam was shone through the lens onto a translucent screen behind which a CCD camera had been placed. The focal length was determined by translating the tunable lens to minimize the size of the spot on the screen. This procedure has been automated (both moving the lens and repeating the process at different drive voltages). 


\section{MODEL OF FOCAL LENGTH VS. VOLTAGE}

In this section, we compute the focal length of a lens as a function of applied voltage on the actuator, for the geometry given in Figure 2, namely one single lens coupled fluidically to one actuator. Since the relation between focal length and membrane vertical deflection is straightforward, this section deals mostly with determining the deflection of a lens when a voltage is applied to the coupled actuator.

Modeling the vertical displacement $z$ of a simple membrane as a function of applied pressure $P$ is based on the Bulge Test equation. The Bulge Test is a technique commonly used to determine the Young's modulus $E$ and initial internal stress $\sigma$ of thin films. Rosset et al. adapted this method to thin PDMS films ${ }^{23}$ The following equation relates $E$ and $\sigma$ of a membrane to the applied pressure $P$ the resulting deflection $z$ of the center of the membrane:

$$
P=\frac{8(1-0.24 v) E t_{0}}{3(1-v)\left(r^{2}+z^{2}\right)^{2}} z^{3}+\frac{4 r^{2} t_{0} \sigma_{0}}{\left(r^{2}+z^{2}\right)^{2}} z
$$

where $v, \varepsilon_{0}$ and $\varepsilon_{r}$ are the Poisson ratio, the vacuum and the relative permittivity; $r$ and $t_{0}$ are the radius and the thickness of the membrane (DEA actuator or lens in our case). The first part of the equation is the stretching induced stress-term and the second is the residual stress induced stress-term.

In [24], Rosset et al. showed that the vertical displacement $z$ of a DEA as a function of pressure $P$ and applied voltage $V$ is obtained by inserting the electrostatic pressure in the previous equation, leading to:

$$
P=\frac{8(1-0.24 v) E t_{0}}{3(1-v)\left(r^{2}+z^{2}\right)^{2}} z^{3}+\frac{4 r^{2} t_{0} \sigma_{0}}{\left(r^{2}+z^{2}\right)^{2}} z-\frac{4 \varepsilon_{0} \varepsilon_{r} V^{2}}{t_{0} r^{2}} z .
$$

Equation 2 applies to the actuator (displacement $z_{D}$ ) and to the lens (displacement $z_{L}$ when $V=0$ ).

We shall assume:

- The total volume $\Lambda$ of fluid inside the device is constant.

- The fluid is incompressible.

- The shape of DEAs and lenses is spherical.

- Gravity is ignored.

- The pressure under the two coupled membranes is identical, $P_{\mathrm{DEA}}=P_{\text {Lens }}$

The physical properties of the DEA and of the lens are known: $E, \sigma_{0}$ are determined with the Bulge Test, $r$ and $t_{0}$ have been measured, and $\varepsilon_{0}$ and $\varepsilon_{r}$ are taken from tables $\left(\varepsilon_{r}=3\right.$ for PDMS). The initial pressure $P_{i}$ is determined from the initial deflection $z_{L i}$ of the lens or $z_{D i}$ of the actuator using Eq. (1).

The objective is to determine $f$ as a function of $V$. This is accomplished by first determining $z_{L}$ vs. $V$ with the following methodology. Apply a voltage $V$ to the actuator, which starts from the initial position $z_{D i}$ and initial pressure $P_{i}$. The actuator membrane moves up (in the orientation of Figure 2, since the area increases) to the new position $z_{D I}$ and pressure drops to $P_{l}$. However from Eq. (2) once cannot uniquely determine $z_{D l}$ and $P_{l}$, but only a curve linking them. The new position of the actuator leads to volume change under the actuator $\mathrm{d} \Lambda_{\mathrm{D}}$, and by conservation of volume to the opposite change in the volume under the lens, $-\mathrm{d} \Lambda_{\mathrm{L}}$. The volume of a spherical cap is given by:

$$
\Lambda=\frac{z\left(r^{2}+z^{2}\right) \pi}{6} \text {. }
$$

Since the pressure is equal on both sides, one can uniquely determine $z_{D I}$ and $P_{l}$ for a given $V$, with $z_{D I}$ given by:

$$
z_{D 1}=r_{D}^{2} \cdot \sqrt[3]{\frac{\pi}{-3 A+\sqrt{9 A^{2}+\pi^{2} r_{D}^{2}}}}-\sqrt[3]{\frac{-3 A+\sqrt{9 A^{2}+\pi^{2} r_{D}^{2}}}{\pi}}
$$

where $A=\Lambda_{\mathrm{Di}}+\Lambda_{\mathrm{Li}}-\Lambda_{\mathrm{L} 1}$, given by Eq. (3). 
Once $z_{D I}$ is obtained (for a given $V$ ), $z_{L I}$ can readily be determined. By numerically and iteratively applying this approach, the lens center height $z_{L l}$ can be determined as a function of voltage.

The focal length $f$ of a plano-convex spherical lens of radius $r$ and thickness $z$ is given by

$$
\frac{1}{f}=(n-1) \frac{1}{R}=(n-1) \frac{2 z}{\left(r^{2}+z^{2}\right)},
$$

where $n$ is the index of refraction and $R$ is the radius of curvature. The optical contribution of the PDMS is ignored, as it is much thinner than the optical fluid. So the focal length of the lens can be computed vs. applied voltage, as a function of initial pressure, geometry, and material characteristics.

Figure 12 is a plot of predicted focal length vs. applied voltage for two different geometries, material properties, and initial pressures, illustrating two possible scenarios, one corresponding to our experimental setup. The focal length can be tuned from its initial value to infinity (flat lens), limited in reality by breakdown voltage and the mass of the liquid.

\section{RESULTS}

The most important phenomena associated with irradiation of polymers are chain scission, chain aggregation, double bonds, molecular emission, cross-linking of the unsaturated adjacent radicals, amorphization of the crystalline fraction of the polymer, and oxidation. ${ }^{25}$ They all influence the elasticity, the internal stress and the optical properties of polymers.

\subsection{Gamma irradiation (Co60)}

\section{Mechanical properties of PDMS vs. Total ion dose (TID)}

The initial values of the Young's modulus and the stress were relatively high due to the applied high curing temperature $\left(150^{\circ} \mathrm{C}\right)$ during the fabrication: $E=2 \mathrm{MPa}$ and $\sigma=60 \mathrm{kPa}$. The results presented in Figure 6 show the relative increase of $E$ and $\sigma$ after gamma irradiation. The values are averaged over 4 lenses for the each dose.

The Young's modulus and the stress increase with the gamma dose. This is due to the irradiation induced chemical changes of the polymer. Also, for the higher dose rate (shorter irradiation time) there is a smaller impact on $E$ and $\sigma$ than the low dose rates. Increasing radiation doses leads to stiffer devices and therefore to reduced actuation.

\section{Optical transmission of PDMS vs. TID}

Transmission properties of the lenses vs. TID are shown in Figure 7. The PDMS membranes are initially highly transparent (>90\%). For the low dose irradiation, a minor decline of transmission can be observed, while at $11.2 \mathrm{kGy}$ an absolute decrease of $20 \%$ is visible. There is no strong wavelength dependence.

\section{Optical transmission of ion-implanted PDMS vs. TID}

Transmission properties of the lenses vs. TID are shown in Figure 8 and it shows that initially DEAs are less transparent than lenses, due to the implantation of both sides of the membrane. The transparency decreases with the gold ion dose. The doses were $1.25 \times 10^{16} \mathrm{at} / \mathrm{cm}^{2}(1), 1.75 \times 10^{16} \mathrm{at} / \mathrm{cm}^{2}(2)$ and $2.25 \times 10^{16} \mathrm{at} / \mathrm{cm}^{2}$ (3). Unlike the lenses (unimplanted PDMS), the DEAs are more affected by gamma irradiation. The largest decrease in transmission is observed for the highest irradiation dose.

In view of the much better transmission (even after $1 \mathrm{kGy}$ irradiation, corresponding to many years operation in most Earth orbits) of non-implanted membranes, we have opted to use non-implanted membranes as lenses, forsaking the flexibility of having the actuators also be lenses for improved optical performance. 


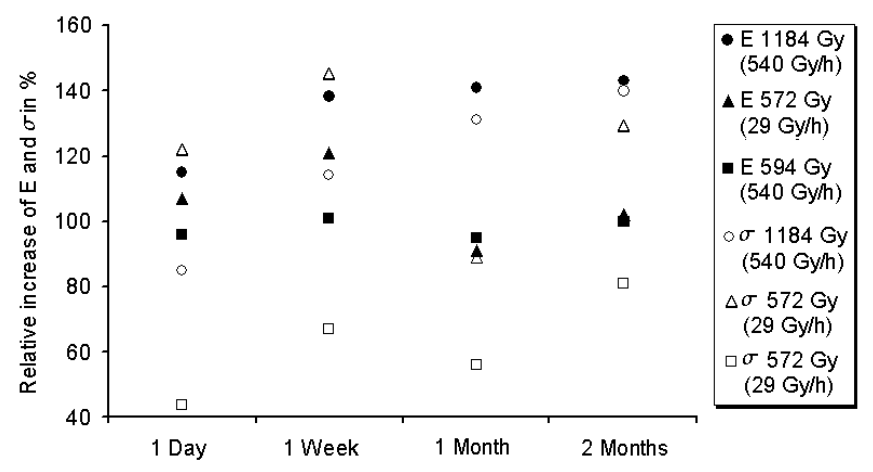

Figure 6. Influence of the gamma radiation on the Young's modulus $E$ and on the stress $\sigma$ of a $30 \mu \mathrm{m}$ thick unimplanted PDMS film as a function of time after irradiation (i.e., as a function of room temperature annealing). Higher gamma doses lead to stiffer membranes.

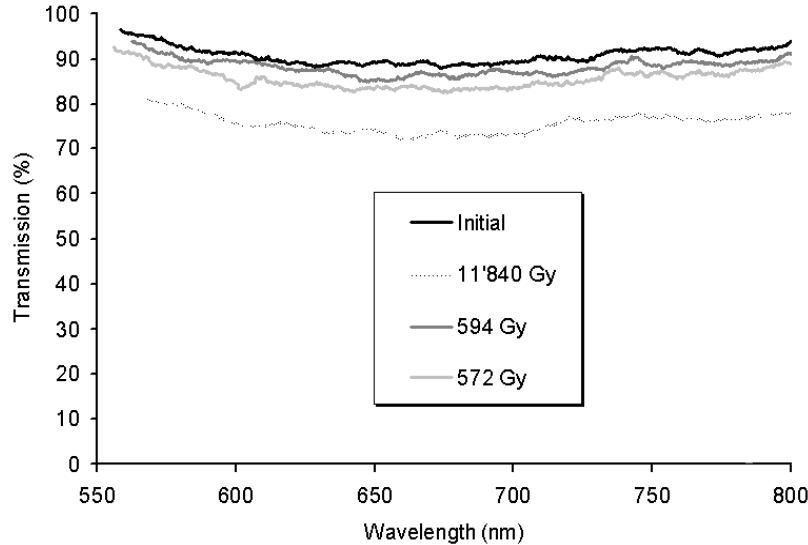

Figure 7. Optical transmission of the unimplanted $30 \mu \mathrm{m}$ PDMS membranes, before and after gamma irradiation. Only at very high doses does optical transmission decrease significantly.

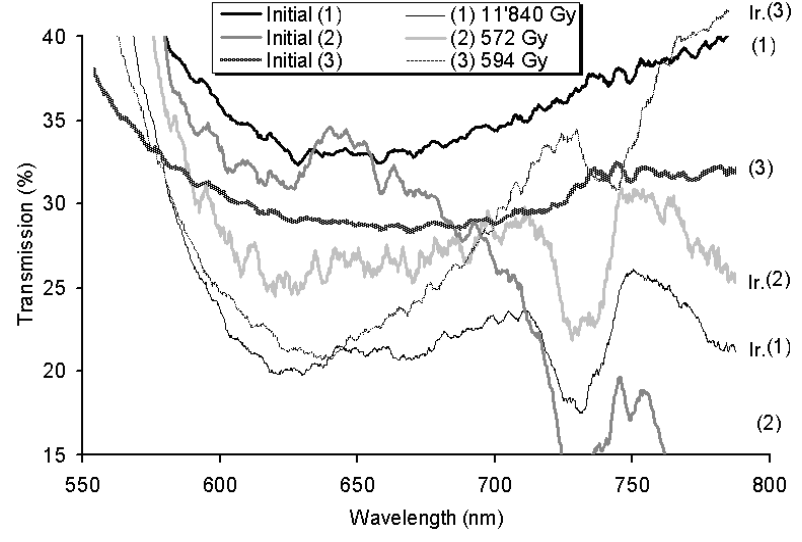

Figure 8. Optical transmission of the implanted PDMS membranes, before and after gamma irradiation, implanted with $\mathrm{Au}$ at the following doses: (1) $1.25 \times 10^{16} \mathrm{at} / \mathrm{cm}^{2}$, (2) $1.75 \times 10^{16}$ at $/ \mathrm{cm}^{2}$, (3) $2.25 \times 10^{16} \mathrm{at} / \mathrm{cm}^{2}$.

\subsection{Proton irradiation (3.5 MeV)}

\section{Mechanical properties of PDMS vs. TID}

The results presented in Figure 9 show the absolute increase of $E$ and $\sigma$ following exposure to $3.5 \mathrm{MeV}$ protons. The values are averaged over 2 membranes for the each dose. Again the Young's modulus and the stress increase with the dose and a higher radiation doses leads to stiffer devices therefore to reduced actuation. Both observations are due to the irradiation induced chemical changes of the polymer.

\section{Optical transmission of PDMS vs. TID}

For the measurements of the optical properties of the proton irradiated lenses and DEAs we used the same testing setup as for the gamma samples. Unlike for the gamma irradiation no important changes of the optical properties were observed for lenses or for DEAs. 


\section{Influence of the plasma $\mathrm{O}_{2}$ on the mechanical properties of PDMS}

We also analyzed the mechanical changes of PDMS following a standard bonding step. The samples were identical to those used for TID tests. They were exposed to $30 \mathrm{~s}$ plasma $\mathrm{O}_{2}$ treatment of the surface - a commonly used procedure for bonding PDMS to glass or PDMS to PDMS. The Young's modulus and the residual stress increased by 33\% and $100 \%$, respectively. One can conclude that this during the fabrication, this necessary step cannot be neglected from the point of view of the mechanical properties. Hence, if very soft membranes are desired, the plasma $\mathrm{O}_{2}$ exposure should be avoided if possible. This can be achieved by clamping the PDMS thin film between two Pyrex chips, instead of bonding it on one chip.

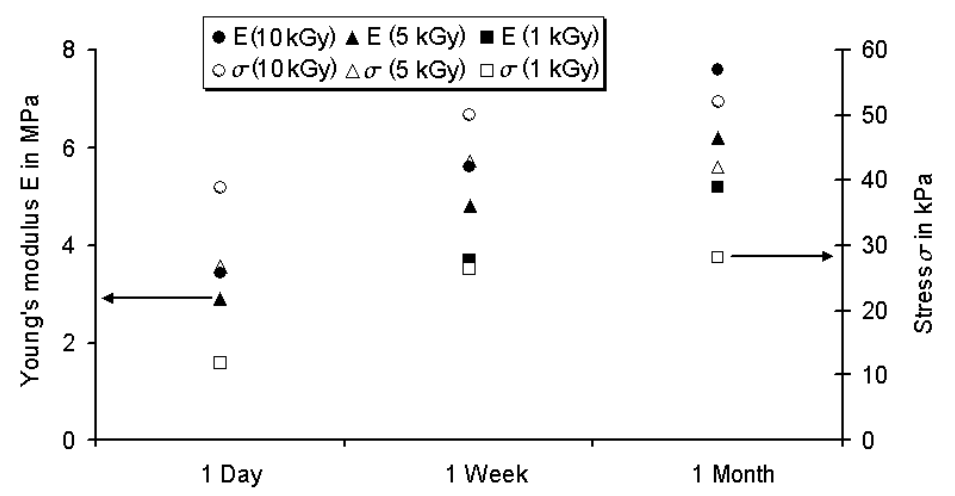

Figure 9. Influence of $3.5 \mathrm{MeV}$ proton radiation on the Young's modulus $E$ and on the stress $\sigma$ of $30 \mu \mathrm{m}$ PDMS membranes (unimplanted). The initial value for $E$ and $\sigma$ were respectively $2 \mathrm{MPa}$ and $60 \mathrm{kPa}$.

\subsection{Performance of the tunable lens device}

A completed (assembled and filled) device is shown in Figure 10, with 4 actuators, which can also serve as lenses. The results of initial testing are shown in Figure 11. A voltage was applied to one DEA, and its deflection, as well as that of the three passive (but coupled) devices was measured.

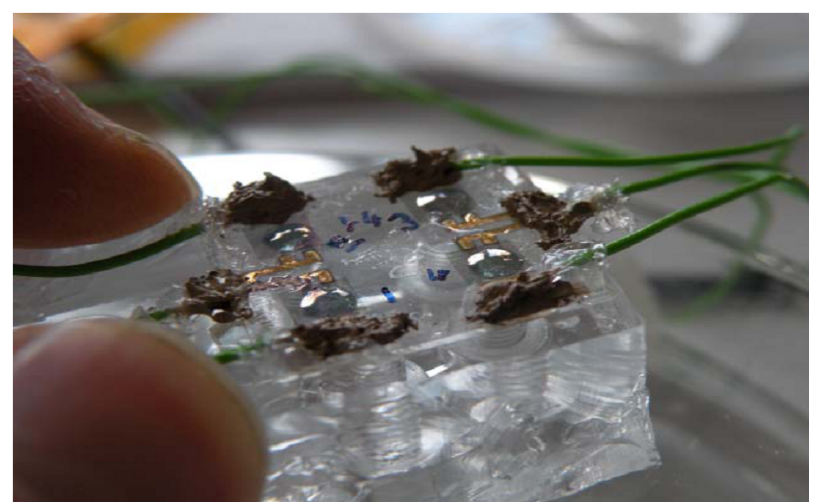

Figure 10. A completed device with a set of four membranes, which can be used as three lenses and one actuator, or one lens and three actuators. For scale, two fingertips are visible on the left.

An actuator deflection of $170 \mu \mathrm{m}$ resulted in a $40 \mu \mathrm{m}$ negative motion of the three lenses. Several tests have been performed for different configurations of the connections between lenses and DEAs. The overall performance of the final device depends on the diameter of membranes and on their mechanical properties, as well as the configuration. 
Device consisting of one lens and one actuator was then tested, with the goal of verifying the model presented in section 4 . The geometry, materials properties $(E, \sigma)$ and lens and actuator deflection $\left(z_{L}, z_{D}\right)$ were measured, as well as the focal length $f$.

The results are presented in Figure 12. The lens focal length varied by a factor of 2 when changing the control voltage from 0 to $1.7 \mathrm{kV}$. For the two tested devices we had similar focal lengths tunable from 4 to $8 \mathrm{~mm}$. The measured actuated and un-actuated focal lengths are in excellent agreement with the values predicted from the model. In Figure 12 the model is the solid line, the data the two circles. There are no free fitting parameters: the

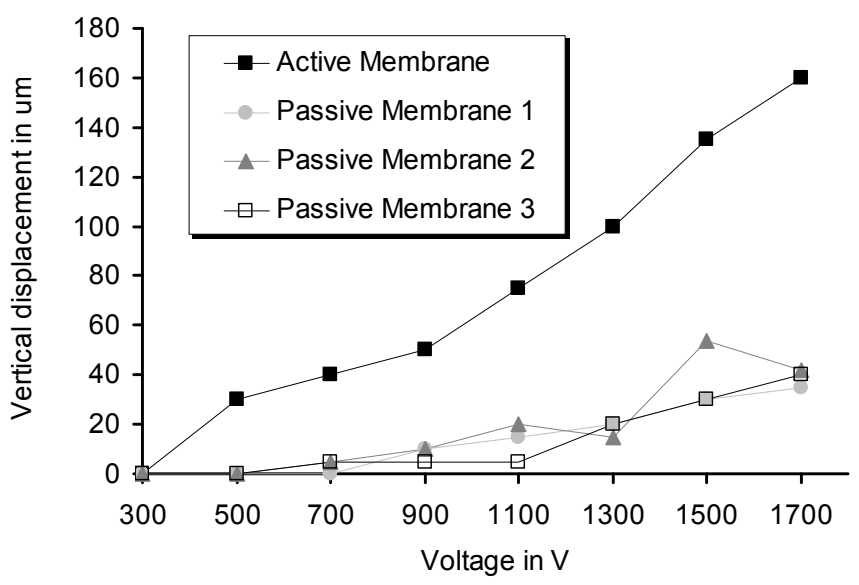

Figure 11. Deformation of the membranes for a water-filled tunable lens assembly (shown in Figure 10). Only one DEA was activated and three membranes respond to the pressure drop inside the device ( 3 lens, 1 actuator configuration). The vertical displacement of DEA is positive (inflating) where it is negative for the lenses (deflating).

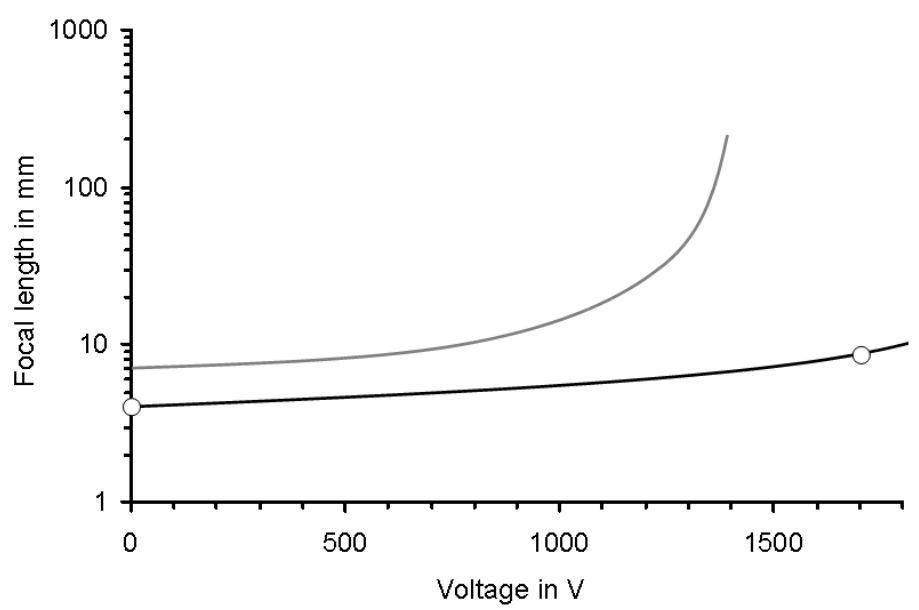

Figure 12. Measured (circles) and predicted focal length (black curve) of a tunable lens device vs. voltage (one actuator and one lens). The properties of the lens are: $E=1.7 \pm 0.1 \mathrm{MPa}, \sigma=62 \pm 0.5 \mathrm{kPa}, r=1 \pm 0.05 \mathrm{~mm}, t_{0}=30 \pm 0.5 \mu \mathrm{m}, P_{i}=3.1 \pm 0.2 \mathrm{MPa}$, $\mathrm{n}=1.33$; the properties of the actuator are: $E=3.9 \pm 0.1 \mathrm{MPa}, \sigma=53 \pm 0.5 \mathrm{kPa}, r=1 \pm 0.05 \mathrm{~mm}, t_{0}=30 \pm 0.5 \mu \mathrm{m}, P_{i}=3.1 \pm 0.2$ $\mathrm{MPa}$. For comparison, the gray curve is the predicted focal length vs. voltage for a device with the following properties (same for lens and actuator) allowing higher tuning range: $E=1 \mathrm{MPa}, \sigma=20 \mathrm{kPa}, r=1 \mathrm{~mm}, t_{0}=20 \mu \mathrm{m}$ and, $\mathrm{P}=500 \mathrm{~Pa}$. Initial focal length and tuning range are a function of geometry, elastomer properties and initial pressure. 
measured properties allow a highly accurate prediction of the focal length. The physical properties of the tested device are given in the figure caption.

The achievable tuning range is limited by the breakdown voltage of the PDMS. Figure 12 presents also the focal length of a single lens coupled with a DEA with different physical properties and an initial pressure of $500 \mathrm{~Pa}$ (gray line). Applying a voltage on the coupled DEA leads to an increase of the lens' radius of curvature, up to the point at which the lens membrane is completely flat, and the focal length diverges to infinity. For membranes with the geometrical and mechanical parameters used for this case, it can be seen on the graph that the chosen initial pressure of $500 \mathrm{~Pa}$ causes the membranes to reach a flat configuration around at $1400 \mathrm{~V}$, well below the breakdown voltage.

The initial pressure set the initial (minimum) focal length. Given a fixed lens diameter, the Young's modulus and thickness of the PDMS must be carefully chosen in order to achieve the desired tuning over the available voltage range. By varying the initial pressure the initial curvature can be set. The PDMS thickness, Young's modulus and stress determine the tuning range, which will be limited by dielectric breakdown.

\section{CONCLUSION}

We have shown the feasibility of tunable polymer micro-optical devices based on ion-implanted polymer actuators. Using a control voltage from 0 to $1.7 \mathrm{kV}$ we demonstrated a tuning range from $4 \mathrm{~mm}$ to $8 \mathrm{~mm}$ for a $2 \mathrm{~mm}$ diameter lens. The model describing the operation of the DEAs and lens interaction has been proposed and shows very accurate results. The tunable lenses operate at high voltage but require almost no power, present high robustness and allow miniaturization and array production. We have shown that the PDMS Sylgard 186 degrades very slowly following high gamma and proton irradiation (over $10 \mathrm{kGy}$ ), increasing in stiffness and loosing optical transparency in a gradual manner, indicating that for most space mission, PDMS-based actuators are much more radiation tolerant than most electronic components. The tunable polymer micro-optical devices bring a new dimension in the field of optics, allowing with further optimization large arrays of individually tunable lenses.

\section{ACKNOWLEDGEMENTS}

We thank Dr. Augusto Tazzoli and the team of Prof. G. Meneghesso at the University of Padova, Italy, for performing the radiation testing. We thank Optotune SA (Zurich, Switzerland) for kindly allowing us to use their focal length measurement apparatus. We acknowledge financial support from the Swiss National Science Foundation grant \#20020-120164, and from the European Space Agency (ESA).

\section{REFERENCES}

[1] C. Friese et al., "Materials, effects and components for tunable micro-optics," IEEJ Trans. Electr. Electron. Eng., vol. 2, no. 3, pp. 232-248, (May 2007).

[2] S. Grilli et al., "Surface-Charge lithography for direct PDMS micro-patterning", Langmuir 24, 13262-13265 (2008).

[3] Y. Hongbin et al., "Simple method for fabricating solid microlenses with different focal lengths", IEEE Photonics Technology Letters, vol. 20, no. 19, 1624-1626 (2008).

[4] X. Zeng et al., "Polydimethlysiloxane microlens arrays fabricated through liquid-phase photopolymerization and molding", IEEE/ASME JMEMS 17, 1210-1217 (2008).

[5] Optotune: www.optotune.com

[6] G.H. Feng and Y.C. Chou, "An eyeball-like biconvex/meniscus lens optical system with fluidic-controlled focus for tunable lens applications", Proceedings of Transducers, Denver, CO, USA, p. 2082 (June 2009).

[7] D.W. Lee and Y.H. Cho, "4-bit digital liquid lens for variable focal length", Proceedings of Transducers 2009, Denver, CO, USA, p.2306 (June 2009).

[8] X. Zeng and H. Jiang, "An endoscope utilizing tunable-focus microlenses actuated through infrared light", Proceedings of Transducers, Denver, CO, USA, p. 1214 (June 2009). 
[9] A. Weber and H. Zappe, “Tunable Pneumatic Microoptics”, IEEE/ASME JMEMS 17, 1218-1227 (2008).

[10] C. Friese and H. Zappe, "Deformable polymer adaptive optical mirrors", IEEE/ASME JMEMS 17, 11-19 (2008).

[11] S. Ashley, “Artificial muscles," Scientific American 289 (4), 52-59 (2003).

[12] Brochu, P., Pei, Q., “Advances In Dielectric Elastomers For Actuators And Artificial Muscles”, Macromolecular Rapid Communications 31, 10-36 (2010).

[13]F. Carpi et al., "Electromechanical characterisation of dielectric elastomer planar actuators: comparative evaluation of different electrode materials and different counterloads," Sensors and Actuators A: Physical 107, 85-95 (2003).

[14] M. Aschwanden and A. Stemmer, "Low voltage, highly tunable diffraction grating based on dielectric elastomer actuators", Proc. of SPIE, vol. 6524 (2007).

[15] R. Pelrine, R. Kornbluh, Q. Pei, J. Joseph,"High-speed electrically actuated elastomers with strain greater than 100\%", Science 287, 836-839 (2000).

[16] S. Rosset et al., "Metal Ion Implantation for the Fabrication of Stretchable Electrodes on Elastomers", Adv. Funct. Materials 19, 470-478 (2009).

[17] R. Kornbluh et al., "Electrostrictive polymer artificial muscle actuators", Proceedings of IEEE Intl. Conference on Robotics and Automation 3, 2147-2154 (1998).

[18] M. Niklaus, et al., "Microstructure of 5 keV Gold implanted PDMS", Scripta Materialia 59, 893-896 (2008).

[19] A. Pimpin et al., "Micro electrostrictive actuator with metal compliant electrodes for flow control applications," presented at MEMS'04 (2004).

[20] S. Rosset et al., "Ion-implanted compliant electrodes for mm-size dielectric elastomer actuators", Proc. of SPIE, Vol. 7287 (2009).

[21] S. Rosset et al., "Large-Stroke Dielectric Elastomer Actuators With Ion-Implanted Electrodes", JMEMS, Vol. 18, 1300-1308 (2009).

[22] S. Rosset et al. "Ion-implanted compliant and patternable electrodes for miniaturized dielectric elastomer actuators", Proc. of SPIE Vol. 6927 (2008).

[23] S. Rosset et al., "Mechanical properties of electroactive polymer microactuators with ion implanted electrodes", Proc. of SPIE, Vol. 6524 (2007).

[24] S. Rosset et al., "Mechanical Characterization of a Dielectric Elastomer Microactuator With Ion-Implanted Electrodes", Sensors and Actuators A 144, 185-193 (2008).

[25] D. Fink, "Fundamentals of ion-irradiated Polymers", Springer, Berlin (2004). 